\title{
The cost of the training and supervision of community health workers to improve exclusive breastfeeding amongst mothers in a cluster randomised controlled trial in South Africa
}

\author{
Gavin George ${ }^{1 *}\left(\mathbb{D}\right.$, Takunda Mudzingwa $^{1}$ and Christiane Horwood ${ }^{2}$
}

\begin{abstract}
Background: Interventions targeting community health workers (CHWs) aim to optimise the delivery of health services to underserved rural areas. Whilst interventions are evaluated against their objectives, there remains limited evidence on the economic costs of these interventions, and the practicality and value of scale up. The aim of this paper is to undertake a cost analysis on a CHW training and supervision intervention using exclusive breastfeeding rates amongst mothers as an outcome measure.

Methods: This is a retrospective cost analysis, from an implementer's perspective, of a cluster randomised controlled trial investigating the effectiveness of a continuous quality improvement (CQI) intervention aimed at CHWs providing care and support to pregnant women and women with babies aged $<1$ year in South Africa.

Results: One of the outcomes of the RCT revealed that the prevalence of exclusive breastfeeding (EBF) significantly improved, with the cost per mother EBF in the control and intervention arm calculated at US\$760,13 and US\$1705, 28 respectively. The cost per additional mother practicing EBF was calculated to be US\$7647, 88, with the supervision component of the intervention constituting $64 \%$ of the trial costs. In addition, women served by the intervention CHWs were more likely to have received a CHW visit and had significantly better knowledge of childcare practices.
\end{abstract}

Conclusion: Whilst the cost of this intervention is high, adapted interventions could potentially offer an economical alternative for achieving selected maternal and child health $(\mathrm{MCH})$ outcomes. The results of this study should inform future programmes aimed at providing adapted training and supervision to CHWs with the objective of improving community-level health outcomes.

Keywords: Costing, Community health workers, Community case management, Continuous quality improvement supervision, Exclusive breastfeeding, South Africa

\footnotetext{
* Correspondence: Georgeg@ukzn.ac.za

${ }^{1}$ Health Economics and HIV and AIDS Research Division (HEARD), University

of KwaZulu-Natal, Durban, South Africa

Full list of author information is available at the end of the article
} 


\section{Background}

Community health workers (CHWs) have the potential to address the barriers encountered by women and children in accessing maternal and child health $(\mathrm{MCH})$ services, particularly in underserved rural areas where the shortage of professional health workers is most profound [1]. CHWs can increase coverage of maternal and child services in African settings, leading to improved maternal and child health outcomes [2, 3]. In 2010, the South African government proposed to re-engineer the provision of primary health care by focusing on a small number of key priorities, including the training and deployment of a generalist CHW that would become a recognised cadre within the national health system [4]. Ahead of implementation of the PHC re-engineering, a 2011 audit estimated that 72,000 CHWs were active across the country [5]. The majority were single purpose workers, linked to NGO-driven HIV and TB programmes offering a plethora of services but without a formal scope of practice.

CHWs focus primarily on community engagement, risk identification, and referral to public health facilities [6]. The comparative advantage of utilising CHWs remains that they are from the community and are therefore closer to the patient and their families and more accessible compared to facility based health personnel $[7,8]$. CHWs can assist in bridging the gaps present in the delivery of integrated care at the health facility level [9] with several studies highlighting the potential role of CHWs in increasing access to health care for individuals living in underserved locations in South Africa [10, 11].

Although CHWs may be considered a less expensive alternative to other professional health cadres, with regard to salary, they should not be seen as a cheap solution for weak health systems as they require support and supervision to be effective and sustainable [1]. Literature has pronounced on this health cadre's lack of supervision as a key impediment to optimising their utility [1, 12], with further studies confirming the lack of support afforded to CHWs by the health system [8] as well as how providers readily offload responsibilities to them without assuming a supervisory role [10]. Many health facility managers similarly have not assumed their supervisory role and provided inadequate support to $\mathrm{CHWs}$ [12]. The effectiveness of CHWs therefore depends on the presence of regular [13] and skilled supervision [14]. Thus, achieving optimal outcomes through the utilisation of CHWs relies on cost-effective strategies that provide quality management and supervision [15].

Research suggests that supervision and constant quality management of the services provided by CHWs will enhance community health outcomes [1, 16, 17]. However, little is known about the costs of such interventions aimed at CHWs. The objective of this study is, therefore, to cost an intervention using the WHO adapted continuous care management (CCM) training materials and a continuous quality improvement (CQI) mentoring and supervision approach targeting CHWs in South Africa [18]. The intervention aimed to achieve a number of objectives as described elsewhere [19]. This costing analysis will express outcomes as natural units represented by the number of women practicing exclusive breastfeeding (EBF) for the first 6 weeks. The number of women practicing EBF is referred to as measuring an outcome expressed in natural units because it describes direct changes in a health state that an intervention introduces [20]. We assume a direct causal relationship between the activities of CHWs and the change in the number of women practicing EBF. EBF is a beneficial practice that reduces incidence of infant and child diarrhoea, hospitalisations for illness, diarrhoea mortality, and all-cause mortality [21]. If implemented globally at near universal levels (>90\%), optimal breastfeeding practices could reduce global child deaths by more than 800,000 , making breastfeeding the most effective preventive intervention to improve infant mortality [21]. Being a beneficial practice, establishing the costs of an intervention aimed at increasing EBF rates would present valuable data for future interventions and programmes aimed at both improving maternal and child health $(\mathrm{MCH})$ outcomes and establishing their cost-effectiveness.

\section{Methods}

\section{Study setting and intervention}

A cluster randomised controlled trial (RCT) investigated the effectiveness of a CQI intervention amongst CHWs providing home-based education and support to selected households, including pregnant women and mothers, in the Ugu Health District in the KwaZulu-Natal (KZN) province, South Africa, between May 2012 and November 2013 [19]. The RCT aimed to measure if the intervention was successful in; 1) improving household caregiving practices including breastfeeding practices, 2) improving uptake of $\mathrm{MCH}$ services, and 3) developing clear referral pathways between the community and the local primary healthcare clinic [19]. The RCT consisted of $150 \mathrm{CHWs}$ randomly allocated to standard of care (SOC) and intervention arms $(n=75$ in each arm). Thirty CHW supervisors were randomly selected from $32 \mathrm{CHW}$ supervisors in the district, and allocated to the intervention or control arms. Four CHWs were randomly selected from the CHWs supervised by each participating CHW supervisor to form a quality improvement team. CHWs were allocated to control or intervention arms based on the allocation of their supervisor. The intervention arm consisted of 15 quality improvement teams comprising five $\mathrm{CHWs}$, including one senior $\mathrm{CHW}$ who functioned as the team supervisor. 
CHWs in the SOC arm received the standard 10-day KZN Department of Health (DoH) training on community-based care for women and infants prior to study initiation. In the intervention arm, $\mathrm{CHW}$ received the standard 10-day KZN DoH training, together with an additional 2-week training on WHO Community Case Management (CCM) prior to the study initiation. CCM training included interactive modules focusing on breastfeeding counselling and supporting breastfeeding in the households during antenatal and postnatal visits, using theory, role-plays and clinical demonstrations to develop CHWs skills. CHWs in the intervention arm also received fortnightly CQI mentoring sessions held over 12 months, these were preceded by a 3-month leadin phase to establish mentoring processes. In addition, quarterly learning sessions took place over the 12-month CQI mentoring period. Quality mentors, who were registered nurses employed by the project and experienced in the use of quality-improvement methods [19], facilitated both the fortnightly mentoring and quarterly learning sessions. This RCT and setting is described in further detail elsewhere [19].

\section{Intervention outcome}

In total, 736 mothers received a $\mathrm{CHW}$ visit at baseline, and 606 at follow up [19]. This cost analysis focuses on mothers of infants $>6$ weeks ( 629 at baseline and 531 at follow up) as illustrated in Table 1.

Overall, mothers served by CHWs allocated to the intervention arm were more likely to receive a visit during pregnancy and the postnatal period. Additionally, mothers served by CHWs in the intervention arm had higher maternal and child health knowledge scores and were more likely to have disclosed their HIV status to the CHW. These results and their significance are detailed elsewhere [19].

\section{Costing}

The aim of this economic evaluation was to compare costs and selected outcomes of this intervention. All costs were identified, measured, and valued from a provider perspective. Data were obtained from project budgets and expenditure reports [22]. Additional data, pertaining to donations were obtained from interviews. In estimating resource use and the economic costs incurred in the RCT, we adopted a direct measurement micro-costing approach [23]. The costing considered both financial and economic costs, where financial costs were the actual expenditures incurred in the purchase of items, and economic costs included the opportunity cost of resource utilisation [23].

Costs are expressed in the final year of the intervention thus, all costs were expressed in 2013 prices, collected in local currency (South African Rands, ZAR) and converted to United States Dollars (US\$) at an average exchange rate in 2013 of US\$1 = R9,66 [24]. Costs were classified as capital or recurrent and captured across three major categories; CHW supportive supervision, $\mathrm{CHW}$ outreach activities and $\mathrm{CHW}$ training. These categories, associated activities and the resource inputs are listed in Table 2.

The costs of the standard 10-day KZN DoH training provided to all $\mathrm{CHW}$ was not included in the analysis. $\mathrm{CHW}$ in the intervention arm who received additional training formed the start-up costs of the intervention and were considered as a capital investment and annualized and discounted, with 10 years chosen as the useful life of the start-up period [25]. This once-off training cost included transport, training venue, accommodation and catering costs which were placed under the category of non-recurrent once-off training costs. The training venue did not carry an explicit cost in the context of the intervention, thus a market price for hiring the venue was obtained and this was included in the cost of the training [26].

All post-training activities were included as recurrent costs and captured in the supportive supervision and outreach categories. This included all costs related to the mentoring and supervision of CHWs. These costed items under mentoring and supervision included personnel, transport, equipment and all other costs related to both mentoring and supervision activities.

Capital costs, within the supportive supervision category included items such as computers and furniture,

Table 1 Reported infant feeding practices amongst participating mothers at baseline and follow-up household surveys

\begin{tabular}{|c|c|c|c|c|c|}
\hline \multicolumn{3}{|l|}{ Baseline } & \multicolumn{2}{|l|}{ Follow up } & \multirow{2}{*}{$\begin{array}{l}\text { Change } \\
\text { OR (95\% Cl) }\end{array}$} \\
\hline & N (\%) & OR $(95 \% \mathrm{Cl})$ & $N(\%)$ & OR $(95 \% \mathrm{Cl})$ & \\
\hline \multicolumn{6}{|c|}{ Amongst mothers of infants $>6$ weeks } \\
\hline \multicolumn{6}{|c|}{ EBF for first 6 weeks of life } \\
\hline Control & $226 / 312(72.4)$ & Reference & $181 / 278(65.1)$ & Reference & \\
\hline Intervention & 207/317 (65.3) & $\begin{array}{l}0.74(0.49-1.1) \\
p \text { value }=0.13\end{array}$ & 194/253 (76.7) & $\begin{array}{l}1.7(1.1-2.7) \\
p \text { value }=0.02\end{array}$ & $\begin{array}{l}2.3(1.4-4.0) \\
p \text { value }=0.001\end{array}$ \\
\hline
\end{tabular}


Table 2 Cost categories and resource inputs for the intervention arm

\begin{tabular}{lll}
\hline Costing Categories & Activities & Resource Inputs \\
\hline Training & WHO Community & Catering \\
& Case Management (CCM) training & Training Venue \\
Support and Supervision & Transport \\
& Fortnightly Mentoring Sessions & Personnel:3 Quality Mentors; 3 Health Facilitators; 1 Administrator \\
Monthly Meetings & Transport \\
& Meeting Venue \\
& Equipment (Laptop, projector \& printer) \\
& Office space \\
Outreach Activities & Utilities \\
& Telephone and data costs \\
& Stationery \\
\hline
\end{tabular}

and other items whose useful life was more than a year. Items whose useful life was more than a year but cost US\$100 or less were classified as recurrent costs [27]. Capital costs were calculated using a discount rate of $3 \%$ and annuitized over a five-year period, as per WHO recommendations [28]. Furniture, including desks, chairs and a filing cabinet, already existed and was utilised and therefore not explicitly purchased for the project, thus indicative costs were obtained from furniture retailers selling similar items. Overhead costs included office rental and utilities. The project was not charged for either, therefore an annual rental and utility cost of the building space was obtained by estimating the annual price charged for renting and expenditure on utilities for a similar sized unfurnished office space [29]. These costs were split across the two arms.

Recurrent costs included labour, which consisted of five personnel types. Three quality mentors, who each received a monthly salary, facilitated the training, supervision and monitored the entire scope of activities undertaken by the CHWs in the intervention arm [18, 19]. Quality mentor's time allocated to facilitating the two-week training represented a small portion of the overall time spent on the project by the quality mentors, with their salaries therefore allocated to the supervision category only. In the control arm, supportive supervision was provided by three health facilitators who were enrolled nurses employed on a full time basis and earning an annual median salary of approximately USD21 305,38 [30] compared to a Quality Mentor earning an annual salary of USD56 236,23. Assuming that the health facilitators allocated $10 \%$ of their time to $\mathrm{CHW}$ activities, it is then estimated that their cost with regards to the control arm is USD2 130, 50 per facilitator. Health facilitators in the control arm met with CHWs on a monthly basis, with no costs incurred. There was one administrator, whose cost was calculated by estimating the percentage of time spent on project related activities multiplied by their full salary [23]. It was estimated that the administrator spent $15 \%$ of their time on project related activities, which was allocated to the support and supervision category [22]. The administrator's salary apportionment was split across the two arms. Other recurrent costs included stationary and supplies, telecommunications and internet data. One quality mentor was provided a cellular phone whilst the other two quality mentors utilised a fixed-line telephone situated in the office. The cost of the fixed-line telephones could not be established. It was assumed that all three-quality mentors used their respective phones equally and thus the cellular phone airtime allowance was used to estimate the fixed-line costs.

CHWs in the intervention arm attended 22 fortnightly mentoring and three quarterly learning sessions, with the costs consisting of a transport allowance paid to $\mathrm{CHWs}$ and venue hire and catering costs. These costs are aggregated to the activity level.

The final category, CHW outreach, encapsulates the home visits undertaken by the CHWs. This category included the stipends paid to CHWs and the cost of supplies utilised by CHWs during home visits. The monthly stipend of the $15 \mathrm{CHW}$ supervisors and $60 \mathrm{CHWs}$ allocated to the intervention arm are represented in the cost analysis.

\section{Cost outcomes analysis}

A cost outcomes analysis compared the costs and outcomes of the control and intervention arm of the study [23]. The aim of this analysis is to determine both the cost per mother practicing EBF, and cost per additional mother practising EBF achieved from the intervention. 
The costs incurred within the training, supportive supervising and outreach categories constitute the intervention costs. A total of 375 mothers had been exclusively breastfeeding for the first 6 weeks of life at the follow-up stage of the study, split into 181 in the control and 194 in the intervention arm (See Table 1). The number of mothers practicing EBF in the intervention arm were adjusted up to account for the larger sample size in the control arm. The percentage of mothers in the intervention arm practicing EBF at follow-up was 76, 7 vs 65,1 of mothers practicing EBF in the control arm. The adjusted number of mothers practicing EBF amongst a total of 278 , with an uptake rate of $76,7 \%$, was calculated to be 213 . The following equation established the cost per additional outcome.

$$
\left(C_{1}-C_{0}\right) /\left(E_{1}-E_{0}\right)=\text { Cost per additional outcome }
$$

$C_{O}$ is the cost per mother practicing EBF in SOC.

$E_{O}$ is the number of mothers practicing EBF in SOC.

$C_{1}$ is the cost per mother practicing EBF in the intervention.

$E_{1}$ is the adjusted number of mothers practicing EBF in the intervention.

\section{Sensitivity analysis}

Studies in similar contexts have used a discount rate of $5 \%$ [31], and $8 \%$ [32, 33]. In acknowledging this variation, a one-way sensitivity analysis was undertaken, using these alternative discount rates [23]. In addition, we undertook sensitivity analysis using a 5 year useful life time horizon for the once-off training. The sensitivity analysis was therefore limited, as other costs were known with a high degree of certainty. A threshold analysis was not undertaken.

\section{Results}

Table 3 presents the costs by input categories. Costs related to $\mathrm{CHW}$ supervision dominated the trial costs, constituting $64 \%$ of all programme costs, followed by CHW outreach activities which contributed $35 \%$ of the total cost.

The greatest proportion of recurrent costs was personnel, contributing over $80 \%$ of the total economic cost of the programme. Quality Mentors make up the highest proportion (44\%) of the costs of the intervention. The Quality Mentors, who were highly skilled professional nurses with management experience, were paid market related salaries. The second highest contributor to costs were the CHW's stipend costs (27\%). The high $\mathrm{CHW}$ personnel costs are a function of the number of CHWs in the programme rather than a reflection of

Table 3 Project costs by category

\begin{tabular}{|c|c|c|c|c|c|c|}
\hline \multirow[t]{2}{*}{ Costing Categories \& Inputs (Quantity) } & \multicolumn{3}{|c|}{ Intervention Arm } & \multicolumn{3}{|l|}{ Control Arm } \\
\hline & Costs (US\$) & $\begin{array}{l}\% \text { within } \\
\text { inputs }\end{array}$ & $\begin{array}{l}\% \text { of total } \\
\text { cost }\end{array}$ & Costs (US\$) & $\begin{array}{l}\% \text { within } \\
\text { inputs }\end{array}$ & $\begin{array}{l}\% \text { of total } \\
\text { cost }\end{array}$ \\
\hline Support Supervision & $244,597,93$ & $100 \%$ & $64 \%$ & $20,433,02$ & $100 \%$ & $15 \%$ \\
\hline Personnel: Quality Mentors (3) / Health Facilitators (3) & $168,708,70$ & $69 \%$ & $44 \%$ & 6391,61 & $31 \%$ & $5 \%$ \\
\hline Administrator (1) & 2739,54 & $1 \%$ & $1 \%$ & 2739,54 & $13 \%$ & $2 \%$ \\
\hline Mentoring Sessions (22) & $51,545,03$ & $21 \%$ & $13 \%$ & - & - & - \\
\hline Quarterly Learning Sessions (3) & $10,302,80$ & $4 \%$ & $3 \%$ & - & - & - \\
\hline Monthly Meetings & - & - & - & 0 & $0 \%$ & $0 \%$ \\
\hline Equipment (Laptop, projector \& printer) & 710,87 & $0 \%$ & $0 \%$ & 710,87 & $3 \%$ & $1 \%$ \\
\hline Office space & 6972,05 & $3 \%$ & $2 \%$ & 6972,05 & $34 \%$ & $5 \%$ \\
\hline Utilities & 1612,32 & $1 \%$ & $0 \%$ & 1612,32 & $8 \%$ & $1 \%$ \\
\hline Telephone and data costs & 747,20 & $1 \%$ & $0 \%$ & 747,20 & $4 \%$ & $1 \%$ \\
\hline Stationery & 1259,42 & $1 \%$ & $0 \%$ & 1259,42 & $6 \%$ & $1 \%$ \\
\hline Once-off Training & 1862,52 & $100 \%$ & $0 \%$ & 0,00 & $0 \%$ & $0 \%$ \\
\hline Combined training costs & 1862,52 & $100 \%$ & $0 \%$ & 0,00 & $0 \%$ & $0 \%$ \\
\hline Outreach Activities & $117,150,62$ & $100 \%$ & $35 \%$ & $117,150,62$ & $100 \%$ & $85 \%$ \\
\hline Personnel: CHWs (60 in each arm) & $89,440,99$ & $76 \%$ & $27 \%$ & $89,440,99$ & $76 \%$ & $65 \%$ \\
\hline Supervisors (15 in each arm) & $26,086,96$ & $22 \%$ & $8 \%$ & $26,086,96$ & $22 \%$ & $19 \%$ \\
\hline Field Materials & 1622,67 & $1 \%$ & $0 \%$ & 1622,67 & $1 \%$ & $1 \%$ \\
\hline TOTAL & $363,611,07$ & & & $137,583,64$ & & \\
\hline
\end{tabular}

Costing Categories and totals in bold 
their skills base. The mentoring and the quarterly learning sessions that CHWs and supervisors attended, contributed a combined $16 \%$ and are the third highest contributor as reflected in Table 3. The once-off WHO adapted CCM training was a negligible cost relative to the total cost of the intervention.

\section{Outputs and costs}

Table 4 illustrates the study outcomes and the associated costs. The cost per mother EBF in the control arm was US\$760,13 and US\$1705,28 in the intervention arm. The cost per additional mother practicing EBF was calculated to be US\$7647,88.

\section{Sensitivity analysis}

Discount rates of 5 , and $8 \%$ were calculated (results not shown). A higher discount rate resulted in a marginally higher cost per additional outcome. Since the change is marginal, the sensitivity analysis indicated that the results were robust to these parameter variations [23]. Additionally, the useful training life was reduced to 5 years from the 10 used in the analysis reported above. Given the relatively low costs attributed to training, this analysis resulted in only a marginal change and is not reported.

\section{Discussion}

This study analysed the costs of implementing a WHO adapted CCM training and a CQI mentoring and supervision intervention, which was successful in increasing EBF rates at 6 weeks post-partum. In this study, the cost per mother practicing EBF in both the control and intervention arms (US\$760,13 and US\$1705,28) is more expensive when compared the cost per additional mother practicing EBF calculated in other South African studies [32], even after adjusting for different outcomes; cost per increased month of EBF [34]. Although the results reveal that the intervention was relatively costly, there were additional benefits accrued, including the increased number of antenatal and postnatal household visits by CHWs and the proportion of mothers with increased health promotion knowledge [19]. These benefits are very likely to have contributed to improved infant feeding practices and care-seeking behaviours of mothers [19]. A unit value could not, however, be allocated to these benefits. If these benefits could be valued, the cost of the intervention could potentially be apportioned over additional outcomes and not solely to EBF, with the resultant costs potentially decreasing significantly, increasing the likelihood of a cost effective intervention.

Carefully selected, trained and supported CHWs offer a potentially economical alternative for promoting and supporting EBF and other key maternal and child health practices to underserved communities, compared with the reliance on overburdened health facilities [21]. Whilst CHWs may potentially be an economical alternative for promoting EBF, CHWs possess less formal training than facility-based health workers. This results in the need for supervision and support [35] such that, to optimise the utility of CHWs, supervision is required [13, 14].The personnel costs associated with skilled supervision are expensive as revealed by this and other studies $[32,34]$, and suggests that the costs of skilled supervision adds substantially to the overall costs of community based programmes. However, without supervision, it is unlikely that optimal benefits will be achieved. In this study supervision was conducted by outside facilitators employed by the study, but in a larger scale rollout it would be possible for existing supervisors to adapt this approach to be used during routine visits to CHWs, thereby reducing costs.

Future research needs to determine whether improved EBF rates, and other $\mathrm{MCH}$ outcomes, remain attainable with different or lower-level health worker cadres operating as supervisors or less costly training or fewer mentoring sessions. It therefore remains crucial to test alternative supervision models aimed at increasing $\mathrm{MCH}$ outcomes using CHWs, whilst costing them. This study therefore adds to a limited body of costing analysis of training and supervisory models targeting CHWs [36].

Further research and analysis on the impact of alternative supervision interventions, combined with an economic evaluation, is essential in assessing whether the added costs are reasonable when compared to the $\mathrm{MCH}$ outcomes achieved [32]. A central tenet of any supervisory model remains scalability and sustainability [19].

\section{Limitations}

The standard 10-day KZN DoH training provided to CHWs in both the control and intervention was not costed. These costs would have affected only the cost per mother practicing EBF result, but not significantly, given the proportion of the cost allocation of the intervention training. Furthermore, the 15 -month time

Table 4 Outcomes and average costs (US\$)

\begin{tabular}{lll}
\hline Outcomes & Total & Average Costs (US\$) \\
\hline Control Arm Mothers Exclusively breastfeeding for first 6 weeks of life & 181 & 760,13 \\
Intervention Arm Mothers Exclusively breastfeeding for first 6 weeks of life (adjusted) & 213 & 1705,28 \\
Additional Mothers Exclusively breastfeeding for first 6 weeks of life & 32 & 7647,88 \\
\hline
\end{tabular}


horizon of the trial may not have been sufficient in terms of realising the study outcomes in their entirety. The initial implementation period of CQI supervision is costly but decreases once CHWs are accustomed to the principles behind CQI management resulting in the tapering off of supervision and the resultant reduction in costs. This highlights the observation that without understanding that supervision is a long-term management intervention, the benefits of CQI may be discounted too hastily [18]. It is important to note that the time between the retrospective cost analysis and the RCT, potentially increased the probability of errors affecting the analysis, primarily due to recall basis [18]. Lastly, this study did not calculate the social costs derived from the increased EBF rates or the opportunity costs of CHWs. These costs are potentially important when determining the true value of an intervention and the outcomes associated with it.

\section{Conclusion}

Whilst CHWs may be a low cost alternative to professional health workers, they require skilled supervision to operate effectively. CHW training and supervision was shown to effectively improve coverage of $\mathrm{CHW}$ visits, improve knowledge of mothers about child care practices, as well as leading to improved household breastfeeding practices. The relevance of this study is that it contributes to research on the cost of providing supervision to CHWs; however, more research around this topic is required.

\section{Abbreviations \\ CCM: Community case management; CHW: Community health worker; CQI: Continuous quality improvement; DoH: Department of Health; EBF: Exclusive breastfeeding; KZN: KwaZulu-Natal; PHC: Primary health care; RCT: Randomised control Trial; US\$: United States Dollars; WHO: World Health Organization; ZAR: South African Rands}

\section{Acknowledgements}

We acknowledge the role of Dr. Jennifer Reddy who was the principal investigator on this trial and director of the 20000 plus partnership at UKZN who tragically died soon after this study was completed. We would like to thank the quality mentors Ellen Ntswe and Khumbusile Sishi for their work with the CHWs.

We wish to acknowledge the input regarding the RCT study design provided by Charles McCulloch, PhD, University of California San Francisco, and statistical analyses provided by Henry Feldman, PhD, Boston Children's Hospital. We would like to thank the KZN Department of Health and Ugu District Management Team for their support. We would also like to acknowledge the community health workers and mothers who participated in this trial.

We appreciate the valuable input of Nigel Rollins, Lyn Haskins, Emma Lansdell and Michael Strauss during the preparation and this manuscript.

\section{Authors' contributions}

GG and CH conceptualised the study. TM assisted with the study design. GG and TM analysed the data. GG prepared the manuscript. All authors edited and approved the final manuscript.

\section{Funding}

Funding was provided by Centers for Disease Control and Prevention (U2G/ PS001081) and World Health Organization (2012/288973).

\section{Availability of data and materials}

The datasets used and/or analysed during the current study are available from the corresponding author on reasonable request.

\section{Ethics approval and consent to participate}

Ethical approval was obtained from Biomedical Ethics Review Committee at the University of KwaZulu-Natal (KZN), World Health Organisation and the University of California, San Franscisco. Permission to undertake the study was obtained from the KZN Department of Health (DoH).

\section{Consent for publication}

Not applicable.

\section{Competing interests}

The authors declare that they have no competing interests.

\section{Author details}

'Health Economics and HIV and AIDS Research Division (HEARD), University of KwaZulu-Natal, Durban, South Africa. ${ }^{2}$ Centre for Rural Health, University of KwaZulu-Natal, Durban, South Africa.

Received: 12 December 2018 Accepted: 16 January 2020

Published online: 03 February 2020

\section{References}

1. Haines A, Sanders D, et al. Achieving child survival goals: potent contribution of community health workers. Lancet. 2007. https://doi.org/10. 1016/S0140-6736(07)60325-0.

2. Pitt C, Tawiah T, Soremekun S, ten Asbroek AH, Manu A, Tawiah-Agyemang C, Hill Z, Owusu-Agyei S, Kirkwood BR, Hanson K. Cost and costeffectiveness of newborn home visits: findings from the Newhints clusterrandomised controlled trial in rural Ghana. Lancet Glob Health. 2016;4(1): e45-56.

3. Mathewos B, Owen H, Sitrin D, Cousens S, Degefie T, Wall S, Bekele A, Lawn $J E$, Daviaud E. Community-based interventions for Newborns in Ethiopia (COMBINE): Cost-effectiveness analysis. Health Policy Plann. 2017;32(suppl_ 1):i21-32.

4. Schneider $\mathrm{H}$. The challenges of reshaping disease specific and care-oriented community based services towards comprehensive goals: a situation appraisal in the Western Cape Province, South Africa. BMC Health Serv Res. 2015;15:1.

5. National Department of Health. Human Resources for Health South Africa. HRH strategy for the Health Sector 2012/13-2016/17. Pretoria: National Department of Health; 2011.

6. Moosa S, Derese A, et al. Insights of health district managers on the implementation of primary health care outreach teams in Johannesburg, South Africa: a descriptive study with focus group discussions. Hum Resour Health. 2017;15:1.

7. Masquillier C. HIV/AIDS competent households: interaction between a health-enabling environment and community-based treatment adherence support for people living with HIV/AIDS in South Africa. PLoS One. 2016;11:3.

8. Loeliger K. "I have to push him with a wheelbarrow to the clinic": community health Workers' roles, needs and strategies to improve HIV care in rural South Africa. AIDS Patient Care STDs. 2016;30:8

9. Uwimana J. Engagement of non-government organisations and community care workers in collaborative TB/HIV activities including prevention of mother to child transmission in South Africa: opportunities and challenges. BMC Health Serv Res. 2012;12:1.

10. Okeyo I, Dowse R. Community care worker perceptions of their roles in TB care and their information needs. Health SA Gesondheid. 2016;21:245-52.

11. Le Roux K. The role of community health workers in the re-engineering of primary health care in rural eastern cape. S Afr Fam Pract. 2015;57:2.

12. Schneider $\mathrm{H}$. Whole-system change: case study of factors facilitating early implementation of a primary health care reform in a south African province. BMC Health Serv Res. 2014;14:1.

13. Bhattacharya $K$, Winch $P$, et al. Community Health Workers Incentives and Disincentives: How they affect motivation, retention and sustainability. Basic Support for Institutionalizing Child Survival Project. 2001.

14. Lehmann U, Sanders D. Community health workers: what do we know about them? The state of the evidence on programmes, activities, costs and 
impact on health outcomes using community workers. Geneva: The World Health Organisation; 2007.

15. Daviaud E, Owen H, Pitt C, Kerber K, Bianchi Jassir F, Barger D, Manzi F, Ekipara-Kiracho E, Greco G, Waiswa P. Overview, methods and results of multi-country community-based maternal and newborn care economic analysis. Health Policy Plann. 2017;32(suppl_1):i6-i20.

16. Schneider $\mathrm{H}, \mathrm{Hl}$ ophe $\mathrm{H}$, et al. Community health workers and the response to HIV/AIDS in South Africa: tensions and prospects. Health Policy Plan. 2008. https://doi.org/10.1093/heapol/czn006.

17. Rahman A, Malik A, et al. Cognitive behaviour therapy-based intervention by community healthworkers for mothers with depression and their infants in rural Pakistan: a cluster-randomised controlled trail. Lancet. 2008;372:902-9.

18. Horwood C, Youngleson M, et al. Using adapted quality-improvement approaces to strengthen community based health systems and improve care in high HIV-burden sub-Saharan African countries. Aids. 2015;29:2

19. Horwood C, Butler $L$, et al. A continuous quality improvement intervention to imporve the effectiveness of community health workers providing care to mothers and children: a randomised controlled trail in South Africa. Hum Res. 2017;15:39.

20. Johannesson M. Theory and methods of economic evaluation of health care. Dordrecht: Kluwer Academic Publishers; 1996.

21. Rollins NC, Bhandari N, Hajeebhoy N, Horton S, Lutter CK, Martines JC, Piwoz EG, Richter LM, Victora CG. Lancet breastfeeding series G: why invest, and what it will take to improve breastfeeding practices? Lancet. 2016; 387(10017):491-504.

22. Center for Rural Health-University of KwaZulu-Natal. Final Draft Report: The Nompilo Project. An evaluation of Community Case Management of childhood illness training and Quality Improvement Supervision for community caregivers to support maternal, neonatal, child and women's health intervention in KwaZulu-Natal, South Africa. Durban: Centre for Rural Health; 2014.

23. Drummond M, Schulpher M, et al. Methods for Economic Evaluation of Health Care Programmes. 4th ed. Oxford: Oxford University Press; 2005.

24. World Bank. South Africa. 2018. http://wdi.worldbank.org/table/4.16. Accessed 12 Dec 2018

25. Baltussen R, Adam T. Generalised cost-effectiveness analysis: a guide. Geneva: The World Health Organisation; 2002.

26. Creese A, Parker D. Cost analysis in primary health care. A training manual for programme managers. Geneva: The World Health Organisation; 1994.

27. Chola L, Fadnes L, et al. PROMISE-EBF study group. Cost-effectiveness of Pear Counselling for the Promotion of EBF in Uganda. PLoS ONE. 2011;10:11.

28. World Health Organisation. WHO Guide to Cost-Effectiveness analysis. Geneva: World Health Organisation; 2003.

29. Drummond $M, O$ 'Brien B. Methods for the economic evaluation of health care programmes. 2nd ed. Oxford: Oxford University Press; 1997.

30. Department of Public Service. Salaries and Benefits in the Public Service. Pretoria: Department of Public Service and Administration; 2016.

31. Gold R, Siegal J, et al. Cost effectiveness in health and medicine. New York: Oxford University Press; 1996

32. Nkonki L, Daviaud E, et al. Costs of promoting exclusive breastfeeding at community level at three sites in South Africa. PLoS One. 2014;9:1.

33. Cleary S, Mclntyre D, et al. The cost-effectiveness of ART in Khayelitsha, South Africa. A primary data analysis. Cost Effect Res Allocation. 2006;4:20.

34. Desmond C, Bland R, et al. Scaling-up exclusive breastfeeding support programmes: the example of KwaZulu-Natal. PLoS One. 2008;3:6.

35. Roberton T, Applegate J, et al. Initial experiences and innovations in supervising community health workers for maternal, newborn, and child health in Morogoro region, Tanzania. Hum Resour Health. 2015;13:19.

36. Hill Z, Dumbaugh $M$, et al. Supervising community health workers in lowincome countries - a review of impact and implementation issues. Glob Health Action. 2014:7:1-10.

\section{Publisher's Note}

Springer Nature remains neutral with regard to jurisdictional claims in published maps and institutional affiliations.

\section{Ready to submit your research? Choose BMC and benefit from:}

- fast, convenient online submission

- thorough peer review by experienced researchers in your field

- rapid publication on acceptance

- support for research data, including large and complex data types

- gold Open Access which fosters wider collaboration and increased citations

- maximum visibility for your research: over $100 \mathrm{M}$ website views per year

At BMC, research is always in progress.

Learn more biomedcentral.com/submissions 